\title{
Review of traditional uses, botany, chemistry, pharmacology, pharmacokinetics, and toxicology of Radix Cyathulae
}

Yongliang Huang ${ }^{1,2+}$, Shanshan Wang ${ }^{2 \dagger}$, Li Liu', Wei Peng ${ }^{2}$, Jiaolong Wang ${ }^{2}$, Ying Song ${ }^{\text {** }}$, Qianghua Yuan ${ }^{1}$, Xing Yuan ${ }^{2}$ and Chunjie $\mathrm{Wu}^{2}$

\begin{abstract}
Cyathulae Radix (CR), also known as "Chuanniux" is a well-known traditional Chinese herbal medicine that has been used in China for thousands of years. The present work reviewed advances in traditional uses, origin, chemical constituents, pharmacology, pharmacokinetics, and toxicity studies of CR. This work aims to provide more up-to-date references for modern study and application of this plant. Furthermore, the possible trends and perspectives for future research of this plant are also discussed. In China, the roots of CR have been widely used in clinical practice to treat orthopedic, gynecological, and urologic diseases. Currently, over 59 compounds have been isolated and identified from CR, including alkaloids and flavonoids. The extracts and compounds from CR have many pharmacological activities both in vivo and in vitro. They provide beneficial effects on the hematological system and anti-inflammatory properties. However, few studies have investigated the pharmacokinetics and toxicity of CR. Further studies should be undertaken to investigate the clinical effects, toxic constituents, and pharmacokinetics of CR; perform quality evaluation; and establish quality criteria for processed C. officinalis. Furthermore, studying the changes of raw and processed CR and the variety of this plant between different cultivated areas and cultivars will be interesting.
\end{abstract}

Keywords: Cyathulae Radix, Traditional usages, Origin, Phytochemistry, Pharmacology, Pharmacokinetics, Toxicology

\section{Introduction}

Cyathulae Radix is a perennial herbaceous plant distributed in Sichuan, Guizhou, and Yunnan provinces in China. Its root is commonly used as a traditional Chinese herbal medicine called "Chuanniux." It is traditionally believed to have the effects of removing "blood stasis," antifertility, anti-inflammation, stimulating menstruation, and curing orthopedic diseases including bone injury [1].

Cyathulae Radix has been listed in the Pharmacopoeia of the People's Republic of China. Since 1953, over 100 prescriptions containing $\mathrm{CR}$ have been utilized to treat gynecological diseases, orthopedic diseases, blood stasis

\footnotetext{
*Correspondence: songying624@163.com

†Yongliang Huang and Shanshan Wang contributed equally to this work

${ }^{1}$ Teaching Hospital of Chengdu University of Traditional Chinese

Medicine, Chengdu 610072, China

Full list of author information is available at the end of the article
}

diseases, stroke, and deficiency of the liver and kidney [24]. CR has various pharmacological activities, including anti-inflammatory effects, effects on the hematological system and immune system, and antihypertensive effects. Many phytochemical studies have been performed on $\mathrm{RC}$, and over 59 compounds have been identified and isolated from this herb, including alkaloids, flavonoids, tannins, triterpenes, and fatty acids. Currently, CR remains a traditional Chinese medicine (TCM) listed in the Chinese Pharmacopoeia, and cyasterone is used as the indicator agent to characterize its quality [1].

In the present paper, the advances in traditional utilization, origin, phytochemistry, pharmacology, and toxicity of CR are systematically reviewed. Relevant literature on CR was collected from Chinese medicine books; articles from Ph.D. and MSc. Dissertations; and scientific databases, including PubMed, ScienceDirect, Web of Science, Springer, Elsevier, Wiley, and CNKI. We systematically reviewed the multi-faceted literature on $\mathrm{CR}$, including 
the origin and advances of phytochemistry, pharmacology, and pharmacokinetics. Furthermore, possible research and application directions and new perspectives on CR are also discussed.

\section{Traditional usages}

Given its wide spectrum of pharmacological activities, $\mathrm{CR}$ has long been used as a medicinal plant in China. In TCM, CR has been used as a wind-damp-dispelling and blood-stasis-removing medicine [5] (Anonymous, 1963). The root of CR is the main part used as a medicine named Chuan Niu Xi. In addition to raw root, CR is commonly processed by stir-baking with yellow rice wine to produce a yellowish or dark brown color. The raw root and wine fried root are the most common clinically used forms [6, 7] (Anonymous, 1977, 2015). The medicinal use of this plant, first listed in Xianshou Lishang Xuduan Mifang in the Tang dynasty in China, dates back to 1170 years ago [8]. In Shennong Bencaojing Shu, the root of CR was described as a herbal medicine with good diuretic and nourishing properties. In the Qing dynasty, another famous TCM monograph Depei Bencao described the root of $C R$ as a herbal medicine with the effects of nourishing the liver and kidney and activating blood to remove stasis. It was described as a treatment for rheumatism, chronic malaria, diarrhea, drench pain, hematuria, stethalgia, and abdominal pain. CR was also described as a medicinal plant used for tonifying the liver and kidney, strengthening the bones and muscles, promoting diuresis, relieving stranguria, and removing blood stasis in other monographs of materia medica in China, including Benjing Fengyuan, Bencao Qiuzhen, and Bencao Zhengyi [3].

Currently, the root of $\mathrm{CR}$ is commonly used as an important TCM for the treatment of various orthopedic diseases (e.g., bone injury, osteoarthritis, rheumatic arthritis, and arthralgia), gynecological disease, and urologic diseases in decoction and proprietary Chinese medicine [3, 6]. In the 2015 edition of the Pharmacopoeia of the People's Republic of China, the root of CR was recorded as a traditional Chinese medicinal material and contained in over 30 prescriptions [9].

\section{Origin}

Cyathulae Radix is a herbaceous perennial plant. It reaches approximately $50-100 \mathrm{~cm}$ in height and contains many branches. The stems of this plant are erect and four prismatic but circular at the bottom. They exhibit green color, which is sometimes mixed with purple. The stems are covered with sparse coarse hairs. The leaves are elliptic to narrowly elliptic, opposite, and petiolate. The leaf margins are entire. The leaves are $3-13 \mathrm{~cm}$ long and
$1.5-5 \mathrm{~cm}$ wide and covered with thick lodging coarse hairs on the pressure side and long pubescence hairs on the reverse side. The flowers have a green-white color and are clustered in inflorescences. The compound cymes gather into flower pellets with a diameter $1-1.5 \mathrm{~cm}$. The flower pellets interactive opposite on rachis. The bisexual flowers are located in the center, whereas sterile flowers are located on both sides. The bracts are ovoid and approximately $4-5 \mathrm{~mm}$ in length. The bisexual flowers are $3-5 \mathrm{~mm}$ in length with lanceolate sepals. The flower has five stamens and five staminodes. The base of the stamen is densely villous. The staminode is rectangular in shape, $0.3-0.4 \mathrm{~mm}$ long, and $0.1-0.2 \mathrm{~mm}$ wide. The pistil is ovary superior and 1-loculed and has a fine style. The utricles are long elliptical, obovate, and $2-5 \mathrm{~mm}$ long. The seeds are ovoid, lentoid, and 1.5-2 mm long. CR flowers from June to July and sets fruit from August to September. The roots of CR are nearly cylindrical, slightly twisted, and 30-60 cm long and have a diameter of $0.5-3 \mathrm{~cm}$. The underpart of the root is slightly slender and has few branches. The surface root is yellowish brown or grayish brown with longitudinal wrinkles, branch marks, and many horizontal long leather-like protuberances. The roots are pliable but strong; they have a light yellow or brown section. The vascular bundles are point-like and arranged in several rounds of concentric rings. The stele is large in the transverse section. The outer tough vascular bundles are intermittently arranged in 4-11 rounds, and the inner stratification layer is visible in the inner vascular bundles. The xylem catheter normally has a single radial arrangement and is ligneous. Wood fiber is developed, and parenchyma cells contain calcium oxalate crystal [1]. Different from other plants of the same genus, the cylinder number of tertiary vascular bundles in 1-year-old CR roots can reach up to six laps, and that in 2-year-old CR roots can reach up to eight laps [10].

This plant is native to South and Southwest China. At present, it is mainly cultivated in Sichuan and Chongqing. The plant is also distributed in some provinces, including Guizhou, Hubei, Hunan, Shanxi, Gansu, Jiangxi, and Zhejiang. The altitude in the cultivation areas ranges from 1150 to $2680 \mathrm{~m}[11,12]$. The plant has two close relatives, C. capitata (Wall.) Moq. and the hybrid of C. officinalis and C. capitata (Wall.) Moq., which are common in origin and widely found in markets. Their roots are identified as adulterant of Chuanniuxi [13]. The main differences between CR and its adulterants are the shape of inflorescence, roots, and taste of the root. Compared with CR, Cyathula capitata (Wall.) Moq. has larger and longer inflorescence and exhibits a taupe color after being dried. The roots are thick, large, and always 
lignified and have an acrid taste. The hybrid of these two species also has a large inflorescence with a black gray color after being dried. The roots taste bitter and cause numb tongue, and they have multiple branches at the bottom. In addition, the duramen of the root is hard [14]. The leaf morphology and microscopic features of these three plants are different [15]. Genetic diversity analysis shows large genetic differences between CR and its adulterants. However, molecular marker technology can easily distinguish them. In 2010, CR and its adulterants were identified by SCAR markers. The primer SC-495 is effective for distinguishing $\mathrm{CR}$ and C. capitata (Wall.) Moq. [16].

\section{Phytochemistry}

Many chemical compounds have been isolated from $\mathrm{CR}$. The chemical components in CR can be roughly divided into three categories: triterpenoid saponins, steroid ketones, and polysaccharide and other compounds (Fig. 1).

\section{Triterpenoid saponins}

Triterpenoid saponins are composed of three saponins and sugar molecules. Tetracyclic triterpenoids and pentacyclic triterpene are common. Sugar that is connected to the glycosides usually contains glucose, rhamnose, glucuronic acid, and arabia sugar. Saponins found in CR are pentacyclic triterpene saponins. Three terpenoid saponins, including oleanolic acid type three terpenoid saponins, ivy saponins, and bamboo saponins, have been reported in CR (Table 1) [17].

\section{Steroid ketones}

Steroid compounds are another important kind of active compounds in CR (Table 2).

\section{Polysaccharide and other compounds}

The polysaccharide of CR is a kind of bioactive polysaccharide extracted from the roots. It has a relative molecular weight of 1000-2200 and is a highly branched fructan. Modern pharmacological studies showed that Achyranthes bidentata polysaccharides can promote cellular immunity and antitumor properties and reduce peripheral leukocyte reduction induced by cyclophosphamide [22]. CR also contains glycine, valine, citramalic acid, ribonic acid, pipecolinic acid, $\mathrm{N}$-carbamylglutamate, gluconic acid, 3-hydroxypropionic, glutaric acid, cellobiose, lactic acid, gluconic acid, xylitol, glutaric acid, pipecolinic acid, ribonic acid, mannose, oxalic acid, digalacturonic acid, lactic acid, 2-deoxyerythritol, acetol, 3-hydroxybutyric acid, $\mathrm{N}$-carbamylglutamate, cellobiose, and palmitic acid [23, 24].

\section{Pharmacology}

Cyathulae Radix possesses extensive pharmacological effects, including effects on the hematological system, urogenital system, and immune system; antifertility effects; anti-inflammatory effects; antitumor effects; senility-delaying effects; hypolipidemic effects; antihypertensive effects; antioxidation effects; and effects on climacteric syndrome, material metabolism, growth and development, and articulation [20]. In the following sections, the primary pharmacological abilities of CR are summarized and analyzed, as listed in Table 3.

\section{Effects on the hematological system}

The characteristic pharmacological ability of CR on the hematological system has been researched on several levels, including the improvement of mesenteric microcirculation, enhancing the deformability of red blood cells (RBCs), reduction of platelet aggregation, decreasing the level of fibrinogen, promotion of wound healing, and lowering blood pressure and blood fat. From the point of view of TCM, CR has the function of activating blood and removing blood stasis. Cheng et al. [25] revealed that the water extracts of RC (WERC) at a dose of $5 \mathrm{~g} / \mathrm{kg}$ could improve mesenteric microcirculation, decrease whole blood viscosity, and strengthen the deformation ability of RBC in acute blood stasis model rats. Wang et al. [26] reported that the addition of CR in Sijunzitang (Decoction of Four Mild Drugs, DFMD) accelerated the healing time and improved the healing rate for refractory wounds in a rat model of lower extremity. The mechanism of these effects is that DFMD increased the expression of vascular endothelial growth factor and the number of microvessels in the wound, which improved the anoxia and ischemia condition, accelerated the healing speed, and shortened the healing time of the wound [26]. After the addition of CR, the effects were stronger than those of DFMD alone. Further investigation revealed that DFMD and CR upregulated the synthesis and secretion of TGF$\beta_{1}$ and $b F G F$, accelerated fibroblast proliferation from the G0/G1 phase to the S phase, promoted the proliferation of fibroblasts and granulation tissues, and filled tissue defects [27]. Moreover, CR increased the proportion of fibroblasts in the S phase. Guo et al. suggested that CR has significant effects on hyperlipidemia and hepatic steatosis. WERC, components of $20 \%$ ethanol elution parts, and steroid components of $\mathrm{CR}$ could reduce the serum TC, TG, LDL-C, ALT, and AST values of hyperlipidemia rats; increase $\mathrm{HDL}-\mathrm{C}$ value; reduce MDA levels in liver tissue; and increase SOD levels.

Moreover, Qi et al. [28, 29] revealed that the ethanol extracts of CR could reduce the blood pressure, decrease the activity of angiotensin-converting enzyme (ACE) and the apoptosis of cardiac cells, and block the effect 

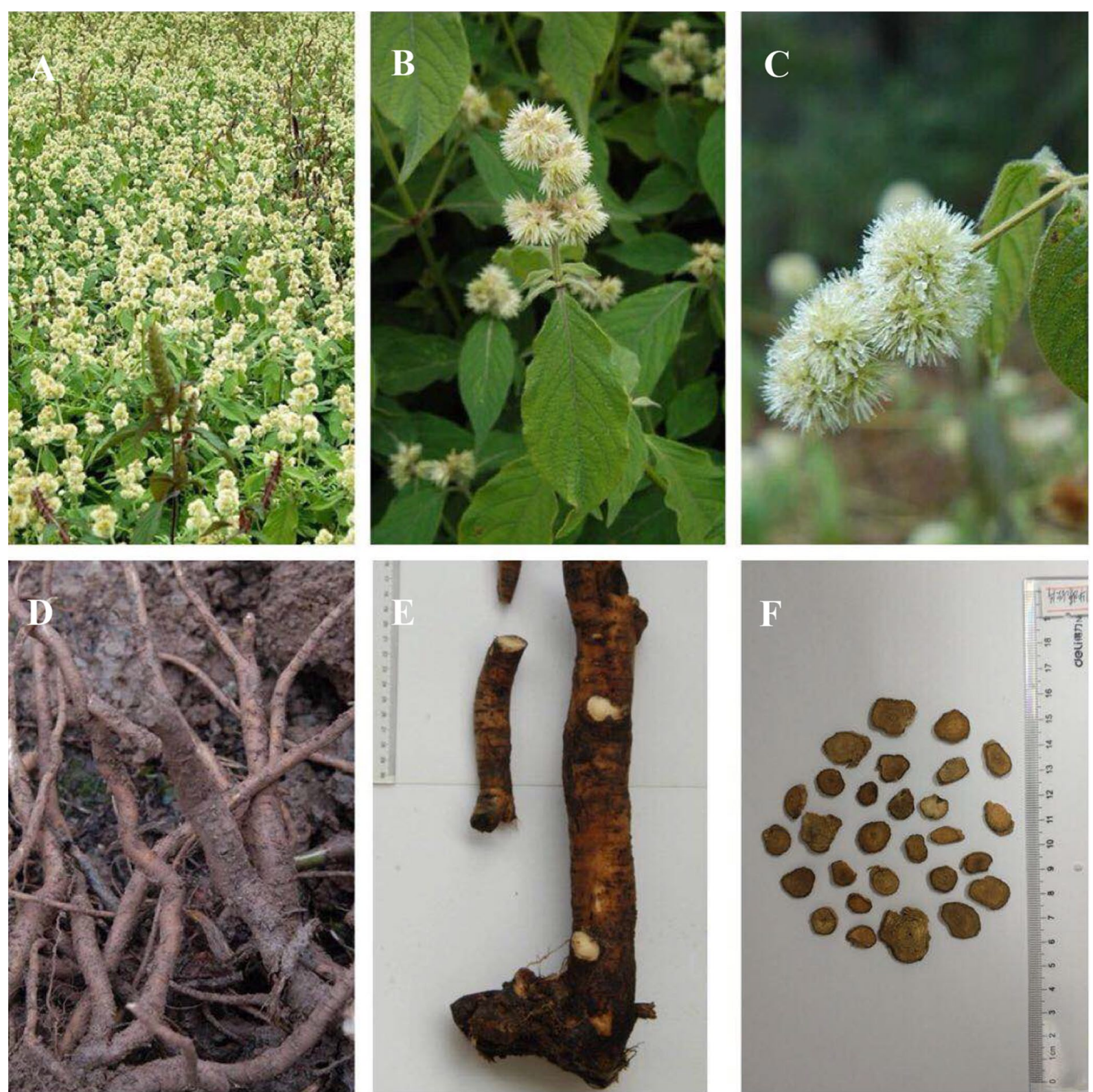

Fig. 1 Cyathulae Radix (A). Flower of Cyathulae Radix (B, C). Roots of Cyathulae Radix (D, E). Transverse section of Cyathulae Radix (F)

of ACE in spontaneously hypertensive rats. In addition, a previous study [30] has also demonstrated that WERC could effectively alleviate left ventricular hypertrophy and improve myocardial remodeling in a dose-dependent manner.

\section{Effects on climacteric syndrome}

Climacteric syndrome in women is due to the decline of ovarian function. Estrogen levels are significantly decreased by cardiovascular, nervous, and endocrine system disorders, leading to dysfunctional uterine bleeding, paroxysmal excess heat and sweating, vulvar and vaginal atrophy, osteoporosis, and other symptoms of the autonomic nervous system. Additionally, estrogen deficiency is the main cause of postmenopausal osteoporosis. Phytoestrogens, an alternative to estrogen, have attracted increasing attention in terms of their safety and efficacy [31].

Wang et al. [32] revealed that CR has a weak estrogenlike effect in ovariectomized rats. It could reduce the atrophy of the reproductive system induced by estrogen deficiency, improve lipid metabolism, and reduce body weight. It may be effective in treating climacteric syndrome. CR could increase uterine weight and improve 
Table 1 Saponins reported from CR

\section{No. Compound name}

3-O- $\beta$-D-glucopyranosyl-( $1 \rightarrow 2$ )-a-L-rhamnopyranosyl-( $1 \rightarrow 3$ )- $\beta$-D-glucopyranosyl-28-O- $\beta$-D-glucopyranosyl oleanolic acid

3-O- $\beta$-D-glucopyranosyl-( $1 \rightarrow$ 2)-[a-L-rhamnopyranosyl-( $1 \rightarrow 3)$ ]- $\beta$-D-glucopyranosyl-28-O- $\beta$-D-glucopyranosyl oleanolic acid

3-O-a-L-rhamnopyranosyl-(1 $\rightarrow$ 3)- $\beta$-D-glucuronopyranosyl-28-O- $\beta$-D-glucopyranosyl oleanolic acid

3-O- $\beta$-D-glucuronopyranosyl-28-O- $\beta$-D-glucopyranosyl oleanolic acid

3-O- $\beta$-D-glucopyranosyl-( $1 \rightarrow 2$ )-a-L-rhamnopyranosyl-( $1 \rightarrow 3$ )- $\beta$-D-glucuronopyranosyl oleanolic acid

3-O-a-L-rhamnopyranosyl-(1 $\rightarrow$ 3)- $\beta$-D-glucuronopyranosyl oleanolic acid

3-O- $\beta$-D-glucuronopyranosyl oleanolic acid

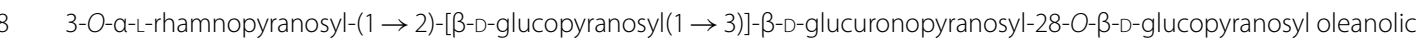
acid

9 3-O-a-L-rhamnopyranosyl-(1 $\rightarrow$ 4)[3-D-glucopyranosyl-(1 $\rightarrow$ 2)]- $\beta$-D-glucuronopyranosyl oleanolic acid or 3-O-a-L-rhamnopyranosyl $(1 \rightarrow 3)[\beta$-D-glucopyranosyl- $(1 \rightarrow 2)]$ - $\beta$-D-glucuronopyranosyl oleanolic acid

10 3-O-a-L-xylopyranosyl-(1 $\rightarrow$ 3)- $\beta$-D-glucuronopyranosyl-28-O- $\beta$-D-rhamnopyranosyl oleanolic acid

11 3-O-[a-L-rhamnopyranosyl-(1-2)- $\beta$-D-glucuronopyranosyl]-28-O-[ $\beta$-D-glucopyranosyl-(1-6)- $\beta$-D-glucopyranosyl] hederagenin

12 3-O- $\beta$-D-glucopyranosyl- $\alpha-L-$-rhamnopyranosyl- $\beta$-D-glucuronopyranosy 1-28-O- - -L-xylopyranosyl hederagenin

13 3-O-Q-L-rhamnopyranosyl-(1-4)- $\beta$-D-glucuronopyranosyl-28-O- $\beta$-D-glucopyranosyl hederagenin or 3-O- $\alpha$-L-rhamnopyranosyl-( $1 \rightarrow 3)-\beta$-D-glucuronopyranosyl-28-O- $\beta$-D-glucopyranosyl hederagenin

14 3-O- $\beta$-D-glucuronopyranosyl-28-O- $\beta$-D-glucopyranosyl hederagenin

15 3-O-Q-D-rhamnopyranosyl-(1 $\rightarrow 3$ )- $\beta$-D-galactopyranosyl-( $1 \rightarrow 3$ )- $\beta$-D-glucuronopyranosyl hederagenin

16 3-O- $\beta$-D-glucuronopyranosyl-28-O-Q-L-rhamnopyranosyl hederagenin

17 3-O- $\beta$-D-glucopyranosyl-a-L-rhamnopyranosyl- $\beta$-D-glucuronopyranosyl-28-O- $\beta$-D-glucopyranosyl gypsogmin

18 3-O- $\beta$-D-glucopyranosyl-( $1 \rightarrow$ 2)-[a-L-rhamnopyranosyl-( $1 \rightarrow 3)$ ]- $\beta$ - D-glucuronopyranosyl gypsogmin

19 3-O-a-L-rhamnopyranosyl- $\beta$-D-glucuronopyranosyl 28-O- $\beta$-D-glucopyranosyl gypsogmin

20 3-O-[a-L-rhamnopyranosyl-(1 $\rightarrow$ 3)-(n-butyl- $\beta$-D-glucopyranosiduronate)]-28-O- $\beta$-D-glucopyranosyl oleanolic acid

21 3-O-[a-L-rhamnopyranosyl-(1 $\rightarrow$ 3)-( $(\beta-D-g l u c u r o n o p y r a n o s y l)]$ oleanolic acid

22 3-O-[ $\beta$-D-glucopyranosyl-(1 $\rightarrow$ 2)-a-L-rhamnopyranosyl-(1 $\rightarrow$ 3)- $\beta$-D-glucuronopyranosyl]-28-O- $\beta$-D-glucopyranosyl oleanolic acid

23 3-O-B-D-glucopyranosyl oleanolic acid

24 28-O- $\beta$-D-glucuronopyranosyl-( $1 \rightarrow 4$ )- $\beta$-D-glucopyranosyl hederagenin

\section{Reference}

Zhou et al. $[18,19]$

Zhou et al. $[18,19]$

Zhou et al. $[18,19]$

Zhou et al. $[18,19]$

Zhou et al. $[18,19]$

Zhou et al. $[18,19]$

Zhou et al. $[18,19]$

Zhou et al. [18, 19]

Zhou et al. [18, 19]

Montoya et al. [20] Montoya et al. [20] Montoya et al. [20] Montoya et al. [20]

Montoya et al. [20] Montoya et al. [20] Montoya et al. [20] Montoya et al. [20] Montoya et al. [20] Montoya et al. [20] Zhou et al. [18, 19] Zhou et al. $[18,19]$ Zhou et al. [18, 19]

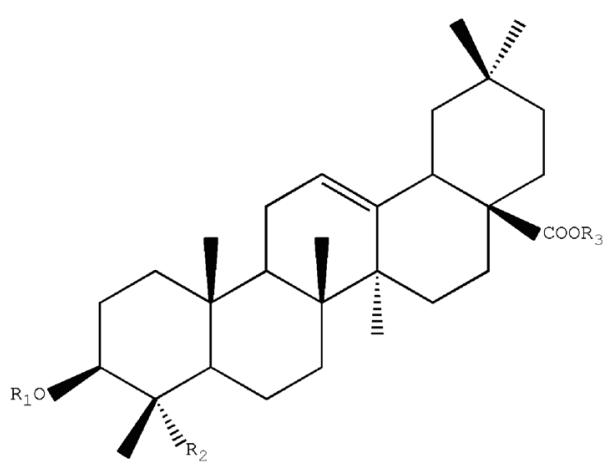


Table 1 (continued)

$\mathrm{R}_{1}$

$\mathrm{R}_{2}$

$\mathrm{R}_{3}$

$\mathrm{CH}_{3}$

$\mathrm{CH}_{3}$

$2 \operatorname{rha}(1 \rightarrow 3) \operatorname{glc} \bigcup^{2}$

1

glc

$3 \operatorname{rha}(1 \rightarrow 3)$ glcUA

4 glcUA

$5 \operatorname{glc}(1 \rightarrow 2) \mathrm{rha}(1 \rightarrow 3) \mathrm{glcUA}$

$6 \quad \operatorname{rha}(1 \rightarrow 3) \operatorname{glcUA}$

7 glcUA

$8 \quad \mathrm{rha}(1 \rightarrow 2) \mathrm{glcUA}$



$\mathrm{CH}_{3}$

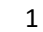

glc

9-1 $\operatorname{rha}(1 \rightarrow 4) \operatorname{glc} \bigcup^{2}$

$\mathrm{CH}_{3}$

$\mathrm{H}$

1

glc

9-2 $\operatorname{rha}(1 \rightarrow 3) \operatorname{glc} \downarrow \mathrm{A}_{2}$

$\mathrm{CH}_{3}$

1

glc

glc
Table 1 (continued)

$\begin{array}{llll}10 & \text { xyl(1-3)glcUA } & \mathrm{CH}_{3} & \text { rha } \\ 11 \text { rha(1-2)glcUA } & \mathrm{CH}_{2} \mathrm{OH} & \text { glc(1-6)glc } \\ 12 \text { glc-rha-glcUA } & \mathrm{CH}_{2} \mathrm{OH} & \text { xyl } \\ 13-1 \quad \text { rha(1-4)glcUA } & \mathrm{CH}_{2} \mathrm{OH} & \text { glc } \\ 13-2 \quad \text { rha(1 } \rightarrow 3) \text { glcUA } & \mathrm{CH}_{2} \mathrm{OH} & \text { glc }\end{array}$

glc

14 glcUA $\mathrm{CH}_{2} \mathrm{OH} \quad$ glc

15 rha $(1 \rightarrow 3)$ gal $(1 \rightarrow 3)$ glcUA $\quad \mathrm{CH}_{2} \mathrm{OH} \quad \mathrm{H}$

16 glcUA $\mathrm{CH}_{2} \mathrm{OH}$ rha

17 glc-rha-glcUA $\mathrm{CHO}$ glc

$18 \operatorname{glc}(1 \rightarrow 2) \operatorname{glcUA}{ }_{3} \quad \mathrm{CHO} \quad \mathrm{H}$

1

rha

19 rha-glcUA

$\mathrm{CHO} \quad \mathrm{glc}$

$20 \quad \operatorname{rha}(1 \rightarrow 3)$ glcUA 6

$\mathrm{H} \quad \mathrm{glc}$

1

n-butyl

$21 \quad \operatorname{rha}(1 \rightarrow 3)$ glcUA

$\mathrm{H}$

$\mathrm{H}$

$22 \operatorname{glc}(1 \rightarrow 2) \operatorname{glcUA}$

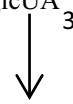

$\mathrm{H}$

glc

1

rha
$\mathrm{H}$

$\mathrm{H}$

$24 \quad \mathrm{H}$ 
Table 2 Phytosterones discovered from CR

\begin{tabular}{lll}
\hline No. & Compound name & Reference \\
\hline 1 & Cyasterone & Zhou et al. $[18,19]$ \\
2 & Isocysterone & Zhou et al. $[18,19]$ \\
3 & 28-Epi-cyasterone & Zhou et al. $[18,19]$ \\
4 & 25-Epi-28-epi-cyasterone & Zhou et al. $[18,19]$ \\
5 & 24-Hydroxycyasterone & Zhou et al. $[18,19]$ \\
6 & Sengosteron & Zhou et al. $[18,19]$ \\
7 & Amaranth A & Zhou et al. $[18,19]$ \\
8 & Precyaterone & Zhou et al. $[18,19]$ \\
9 & Makisterone B & Zhou et al. $[18,19]$ \\
10 & 2,3-Isopropylidene cyasterone & Zhou et al. $[18,19]$ \\
11 & 2,3-Isopropylidene isocyasterone & Zhou et al. [18, 19] \\
12 & Ecdysterone & Zhou et al. [21] \\
13 & 25R Inocosterone & Zhou et al. [21] \\
14 & 25S Inocosterone & Zhou et al. [21] \\
\hline
\end{tabular}

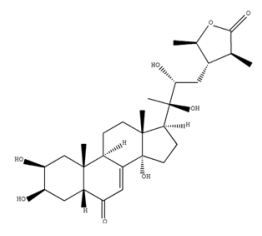

1. Cyasterone

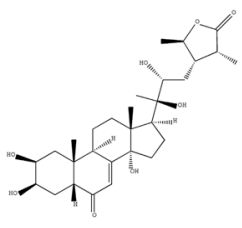

2. Isocysterone

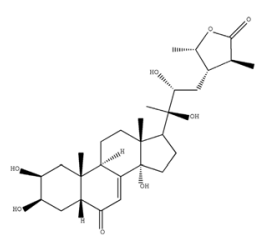

3. 28-epi-cyasterone

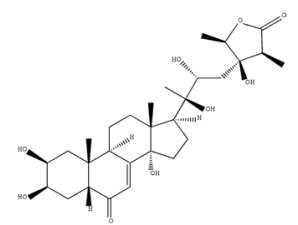

5. 24-hydroxycyasterone



4. 25-epi-28-epi-cyasterone

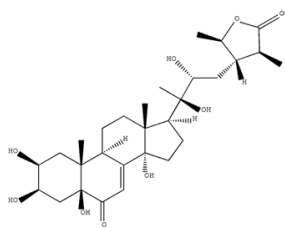

6. Sengosteron
Table 2 (continued)



7. 24-hydroxycyasterone

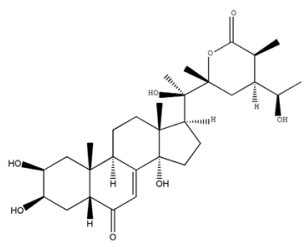

8. Sengosteron

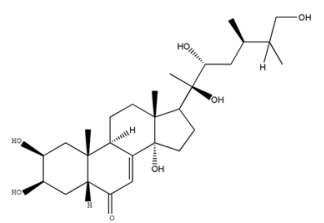

9. 24-hydroxycyasterone

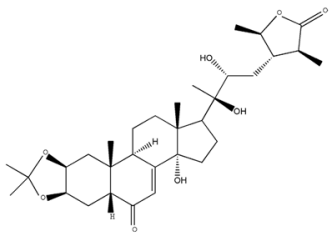

10. Sengosteron

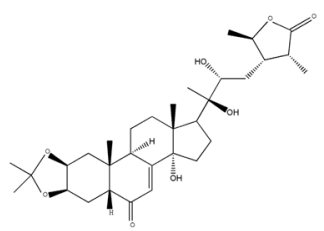

11. 24-hydroxycyasterone



12. Sengosteron

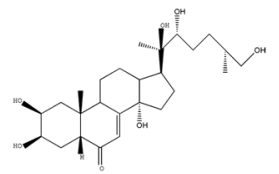

13. 24-hydroxycyasterone<smiles></smiles>

14. Sengosteron

endometrial atrophy in ovariectomized rats, indicating that it has the same function with endogenous estrogen. However, its stimulating effect on the endometrium is far less than that of estrogen. So the treatment of postmenopausal estrogen deficiency by using RC instead of estrogen can avoid the risk of inducing endometrial and breast cancer. Furthermore, CR shows a notable inhibitory 


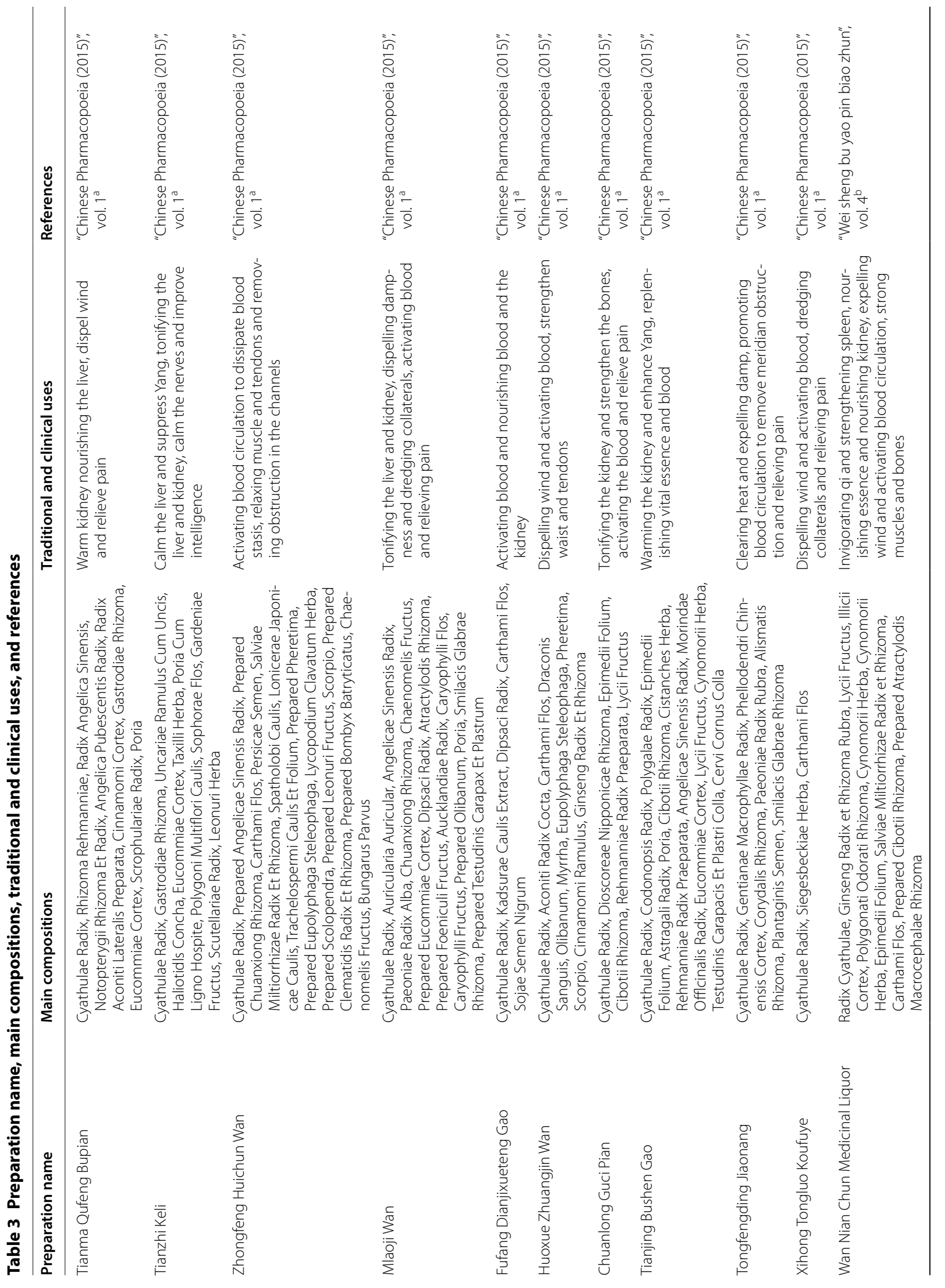














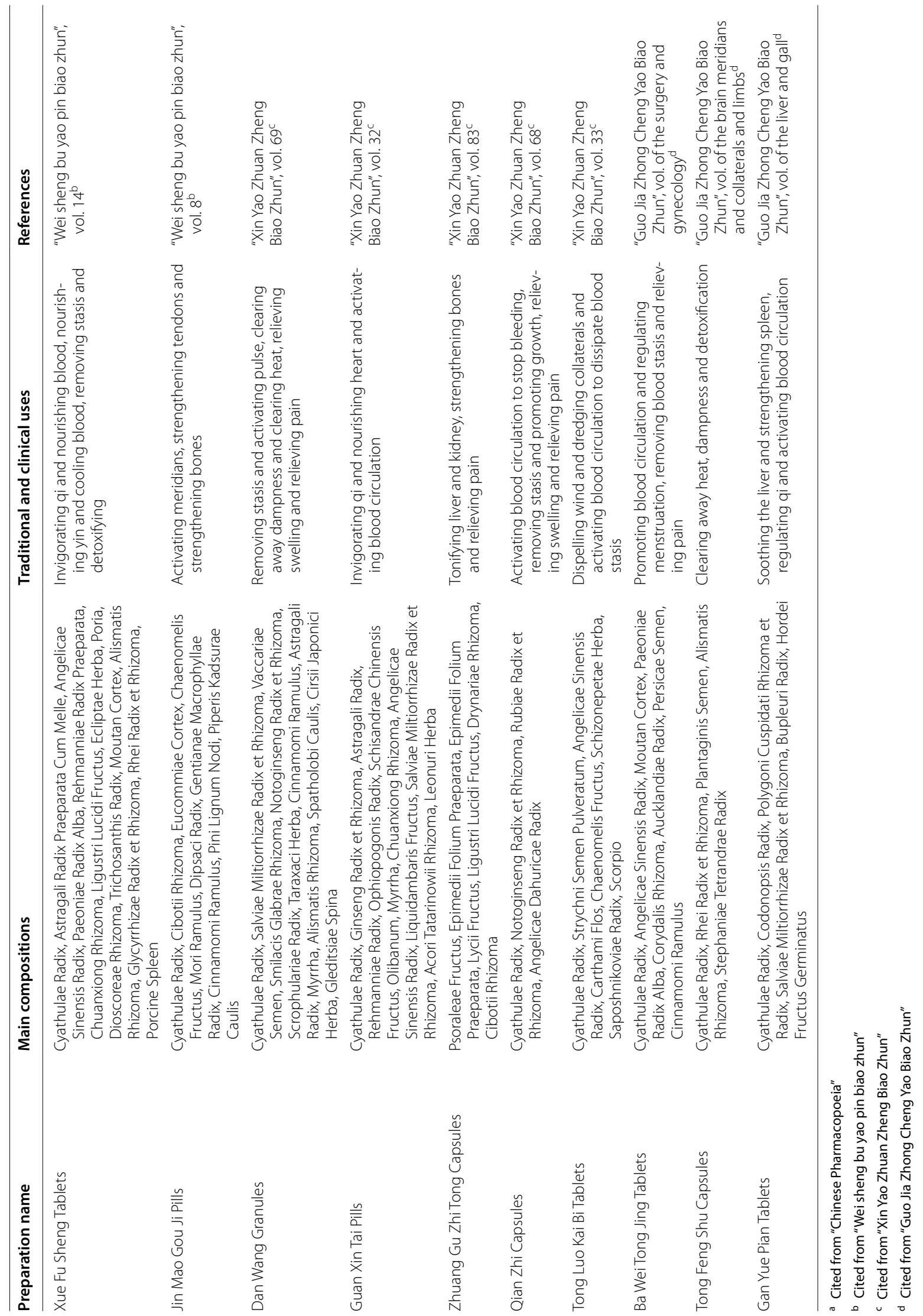


effect on bone loss, improving bone biomechanical properties and preventing the occurrence of osteoporosis in ovariectomized rats.

In addition, $C R$ reduces the degree of joint edema in rats with acute ventilated arthritis. The mechanism of the effect may be associated with downregulating the expression of NF-kB P65 and inhibiting the activity of NF-kB, thereby reducing inflammation [33].

\section{Effects on the immune system}

Polysaccharides of CR (PCRs) possess adjuvant potential on cellular and humoral immune responses. Feng et al. found that PCRs $(25,50,100 \mu \mathrm{g} / \mathrm{mL})$ significantly enhanced the phagocytic capacity of peritoneal macrophages; splenocyte proliferation; and the activity of natural killer cells, cytotoxic T lymphocytes, and OVAspecific IgG, IgG1, IgG2a, and IgG2b antibody titers. Furthermore, PCRs promoted the levels of interleukin-2 (IL-2), interferon (IFN)-, and IL-4 in CD4(+) T cells and IFN- in CD8(+) T cells. In addition, PRCs enhanced the expression of $\mathrm{CD} 40(+)$, $\mathrm{CD} 80(+)$, and $\mathrm{CD} 86(+)$ in dendritic cells (DCs). More importantly, PCRs downregulated the frequency of CD4(+)CD25(+)Foxp3(+)Treg cells. Taken together, these results suggested that PRCs could increase cellular and humoral immune responses by upregulating DC maturation and suppressing Treg frequency [34].

PCRs have attracted increasing attention from researchers. Li et al. [35] found that PCRs $(2.5,5,10 \mathrm{~g} / \mathrm{kg})$ played a key role in enhancing the PFC response ability of mice immunized with SRBC, strengthening the cytotoxic activity of NK cells. However, the lymphocyte transformation rate did not change significantly. This finding showed that PRC could enhance the role of humoral immunity mediated by B cells, had no significant effect on $\mathrm{T}$ cell-mediated immunity, and could enhance the cytotoxic activity of NK cells. Wang et al. [36, 37] discussed the immune function of Achyranthes bidentata polysaccharides in vivo and in vitro. In vivo, PCR (25-100 mg/ $\mathrm{kg}$ ) could enhance delayed hypersensitivity and NK cell activity in normal mice and increase the carbon clearance rate in mice, number of antibody-producing cells, and macrophage phagocytosis of chicken RBCs with increasing polysaccharide concentrations. PCR could also significantly improve the number of white blood cells caused by cyclophosphamide (Cy) reduction. However, it had no effect on the transformation rate of spleen lymphocytes. In vitro, PCR in the concentration range of $10-300 \mu \mathrm{g} /$ $\mathrm{mL}$ did not have a direct toxic effect on cells. It could promote the proliferation of $\mathrm{B}$ lymphocytes $(\mathrm{P}<0.01)$ and enhance NK cell activity in mice peritoneal macrophages $(\mathrm{P}<0.05)$ and neutral $\mathrm{RBC}$ activity $(\mathrm{P}<0.01)$ with increasing polysaccharide concentration. However, it had no effect on the proliferation of $\mathrm{T}$ lymphocytes $(\mathrm{P}>0.05)$. In the theory of TCM, CR possesses a tonic effect, which may be related to its polysaccharides.

\section{Antitumor effect}

Song et al. [38] revealed that PCR has a certain inhibitory effect on mouse hepatoma cells. Ding [39] reported that PCR at doses of $1,2.5$, and $5 \mathrm{~g} / \mathrm{kg}$ could effectively suppress mouse $\mathrm{H} 22$ hepatoma cells with inhibition rate of $21.99-42.21 \%$. In S180 tumor-bearing mice, the inhibition rate was $10.00-48.08 \%$ with PCR dose of 1 and $5 \mathrm{~g} /$ $\mathrm{kg}$. PCR not only had strong antitumor effects but also played a significant role in the recovery of peripheral leukocytes from mice (normal and tumor-bearing mice) induced by Cy. Zong et al. [40] revealed that 100, 200, and $400 \mu \mathrm{g} / \mathrm{kg}$ of PRC inhibited the growth of BEL-7402 cells and promoted cell apoptosis. The mechanism might be related to the upregulation of caspase- 3 protein expression. Cao et al. [41] studied the effects of PCR on the tumor microenvironment of mice bearing HepG-2 cells. They administered $0.825 \mathrm{~g} / \mathrm{kg}$ per day (low dose), $1.65 \mathrm{~g} /$ $\mathrm{kg}$ per day (middle dose), and $3.3 \mathrm{~g} / \mathrm{kg}$ per day (high dose) of PCR in mice by gavage for 2 weeks. The results showed that PCR improved tumor immune inhibition, and high dose of PCR inhibited the expression of immunosuppressive factors in local tumor tissues and delayed tumor progression.

\section{Antioxidation and antiaging effects}

Ding et al. [42] established a rat model of hepatocyte injury induced by $\mathrm{H}_{2} \mathrm{O}_{2}$ in vitro. They treated rats with $10 \mathrm{mg} / \mathrm{mL}$ of PCR with $73 \%$ purity. The results showed that PCR could reduce the increased levels of aminotransferase induced by $\mathrm{H}_{2} \mathrm{O}_{2}$ in rat stem cells, which might be related to the antioxidant effect of PCR. In a senescent mouse model, $0.25 \mathrm{~mL}$ of $55.6 \%$ pure Achyranthes bidentata polysaccharide was intragastrically administered, and $0.2 \mathrm{~mL}$ of D-galactose was injected subcutaneously [43]. The results showed that A. bidentata polysaccharides had stronger antioxidant activity than vitamin $C$, which is an excellent natural oxidant.

\section{Effects on material metabolism and growth}

Cheng et al. $[44,45]$ revealed that CR contains molting sterones. Molting steroids increase glucose consumption within a certain concentration range, and the hypoglycemic effect of molting sterones decreases with increasing glucose concentration in the culture medium. The hypoglycemic effect of the molt is associated with hepatocytes and is non-insulin dependent. In a cell model of insulin resistance, ecdysterone can increase insulin sensitivity and improve glucose metabolism. As a reverse inhibitor, 
ecdysterone can improve insulin resistance caused by hyperglycemia, and its toxicity is very negligible.

\section{Effects on promoting angiogenesis}

Cyathulae Radix is believed to stimulate menstruation and affect bone injury. Angiogenesis is actively involved in treating these illnesses. Zhou et al. reported that the whole extract of CR evidently increased cell migration. The open wound area was smaller than that of the control group by $14.9 \%$ after an $8 \mathrm{~h}$ treatment. The whole extracts of CR promoted angiogenesis mainly via stimulating the migration of endothelial cells in human umbilical vein endothelial cells in vitro. The authors found that several migration-related genes in zebrafish appeared to play pro-angiogenic roles of the CR extract in vivo. The results in human ECs are quite comparable to those in zebrafish. The mechanism of these effects is related to the enhanced gene expression of MMP9 and $\beta$-catenin by CR extract, revealing that matrix degradation and cell-cell adhesion are simultaneously triggered by the CR extract for stimulating angiogenesis. $\beta$-Catenin is a critical member of the canonical Wnt signaling pathway that is responsible for vascular sprouting, particularly for cell-cell adhesion. Moreover, it can trigger signal transduction for vascular remodeling and differentiation [46].

\section{Pharmacokinetics}

To date, pharmacokinetic studies of CR extracts are few. Previous studies mainly focused on its ketosteroids such as cyasterone. In 2010, a study [47] reported that cyasterone is abundant in CR and can be used as a representative ingredient in pharmacokinetic studies. A rapid and sensitive method using reverse phase high-performance liquid chromatography (HPLC) was established to determine the cyasterone concentrations in rabbit plasma. The method provided bases for the study on CR pharmacokinetics. CR was traditionally considered as a guiding herb in TCM composite preparations and deeply valued by tens of millions of Chinese medicine practitioners. Tang et al. studied the effects of CR on paeoniflorin pharmacokinetics of Xuefu Zhuyu Tang in normal and blood-stasis model rats. After oral administration of solution with or without CR at a dose of $5 \mathrm{~mL} / \mathrm{kg}$, the peak plasma concentration (Cmax), peak time (Tmax), and half-life values of paeoniflorin were determined. The results indicated that the presence of CR in Xuefu Zhuyu Tang increased the Cmax of paeoniflorin from 0.099 to $0.363 \mathrm{mg} / \mathrm{L}$ and 0.228 to $0.315 \mathrm{mg} / \mathrm{L}$ in the normal and blood-stasis model group, respectively. Moreover, CR decreased the Tmax from 0.555 to $0.276 \mathrm{~h}$ in the normal group and the $\mathrm{t} 1 / 2$ from 0.853 to $0.501 \mathrm{~h}$ and 0.708 to $0.408 \mathrm{~h}$ in the two groups. Furthermore, the area under the concentration-time curve (AUC) was also determined. AUC0- $\infty$ increased from 0.166 to $0.381 \mathrm{mg} / \mathrm{L} \mathrm{h}$ in the normal group only. As a guiding herb, RC could significantly increase the absorption amount and rate of paeoniflorin in decoction and accelerated its elimination from the blood [48].

\section{Toxicity}

Throughout its long history, CR has been generally considered to be a safe TCM in China [49]. No reports of poisoning have been reported in clinics. In a previous study, the toxicity of its ingredients such as ecdysone was found to be very low. The embryotoxicity of CR was also mentioned in the literature; however, a study on mice showed that its water decoction had no obvious induction effect of chromosomal aberration and embryonic micronucleus [50]. Recently, the acute toxicity of CR was reported in a dissertation [51]. The experimental results showed that after high-dose administration of water decoction, the mice exhibited neurotoxicity and gastrointestinal toxic reaction. Obvious toxic reactions were observed. Moreover, the maximum tolerance dose of samples from different origin ranged from 56.8 to $34.9 \mathrm{~g} /$ $\mathrm{kg}$. This experimental result was different from the traditional cognition of safety of CR. However, the toxicity has not been clarified to date. Thus, further studies should be conducted to confirm the clinical innocuity of CR.

\section{Future perspectives and conclusions}

CR has been used in China as an effective TCM for many years. Recently, it has been the subject of increasing interest. Many kinds of chemical constituents have been isolated and identified from this plant, and the pharmacological activity of its main chemical constituents such as alkaloids, steroids, and polysaccharides has been verified. Meanwhile, many traditional uses of this plant have been validated by pharmacological studies. In addition, previous animal investigations and in vitro studies have revealed that $C R$ possesses significant effects on the hematological, urogenital, and immune systems. There is no doubt that continued progress has been made on various aspects of this plant in the past decade. However, further preliminary studies are needed to fully elucidate the mechanisms and constituents of this plant.

First, due to the complex introduction process, the mixture of CR and its adulterants has been widely used. This process has led to the common occurrence of adulterants in the herbal medicine market and misapplications in clinics, thus significantly affecting the value of CR. Therefore, cultivation management should be strengthened to remove the adulterants from the cultivation base. In addition, a unified identification is needed to control the variety and quality of this herbal medicine, and establishing a standardized finger print of this plant might be indispensable. 
Second, the current pharmacological studies of this plant have primarily focused on several chemicals such as cyasterone and achyranthan. Evidence that can explain the special mechanism of action for the pharmacological activity of this plant is lacking. Thus, future studies should investigate more bioactive compounds and their mechanism of action and structure-function relationship. In addition, toxicity studies of aqueous extract are few, and no evaluations of target-organ toxicity have been documented. Furthermore, the experimental results about neurotoxicity and gastrointestinal toxicity contradict traditional understanding of CR. Therefore, further studies should devote more effort to investigate the compounds of $C R$ and their toxicity.

Third, studies regarding the pharmacokinetics of CR are lacking. According to the only pharmacokinetic report of this plant, the marker compound cyasterone could be determined accurately in rabbit plasma via HPLC. Additionally, as a guiding-herb, CR could affect the pharmacokinetics of other medicines in Chinese compound formula. Therefore, future studies should investigate the pharmacokinetics of its main compounds.

Fourth, to date, studies on the parts of CR other than its roots are few. Other parts of this plant are worthy of multi-aspect research in the future. Thus, the chemical constituents and pharmacological effects of the aboveground parts should be investigated to reveal their possible medicinal potential.

Fifth, the traditional uses of CR include several processed products including the roots stir-fried with wine and salt. The traditional theory considered that the processing techniques with wine and salt could enhance its function of promoting menstruation and blood circulation and strengthening the muscles and bones [52]. Current investigations have found that the two processing techniques have significant effect on herbal chemical profiles, and several new compounds are generated in the process [53]. However, there is still not enough research to confirm the change of efficacy in the process. Moreover, special quality standards for processed CR are still lacking. Therefore, studies should investigate the chemical and pharmacological characteristics of processed CR systematically. Lastly, although CR is currently widely used and cultivated in many provinces of China, managing the variety of this plant and enhancing fundamental research are also important.

In addition, a similar Chinese medicine, Achyranthis Bidentatae Radix which is named Niuxi or Huainiuxi is used in the clinic. The original plants of Achyranthis Bidentatae Radix (Niuxi) and Cyathulae Radix (Chuanniuxi) looked similar and both of them are used to invigorate the circulation of blood. However, the main difference between these two herbs is efficacy. Achyranthis Bidentatae Radix is more suitable for tonifying the kidney and strengthen the muscles and bones, while Cyathulae Radix prefers promoting blood circulation and removing blood stasis, according to Chinese Pharmacopoeia 2015 Edition and teaching materials. Although this distinction is controversial now [54], it is used clinically according to this distinction.

Our present paper has systematically reviewed the traditional uses, origin and variety, phytochemistry, pharmacology, and toxicology of CR and provided comprehensive information on this plant. We hope that this review highlights the importance of $\mathrm{CR}$ and provides some directions for the future development of this plant.

\section{Abbreviations \\ CR: Cyathulae Radix; HPLC: high-performance liquid chromatography.}

\section{Authors' contributions}

YH wrote the Introduction and Traditional usages sections; SW wrote the Phytochemistry and Pharmacology sections; LL wrote the Origin section; JW wrote the Pharmacokinetics section; XY wrote the Toxicity section; and QY wrote the Future perspectives and conclusions section. WP finalized the draft. YS and CW designed the whole structure of the manuscript. All authors read and approved the final manuscript.

\section{Author details \\ ${ }^{1}$ Teaching Hospital of Chengdu University of Traditional Chinese Medicine, Chengdu 610072, China. ${ }^{2}$ Chengdu University of Traditional Chinese Medi-} cine, Chengdu 610075, China.

\section{Acknowledgements}

Not applicable.

\section{Competing interests}

The authors declare that they have no competing interests.

Availability of data and materials

The datasets used and/or analyzed during the current study are available from the corresponding authors on reasonable request.

Consent for publication

Not applicable.

Ethics approval and consent to participate

Not applicable.

Funding

This work was supported by the National Standardization Project of Traditional Chinese Medicine (No. 2YB221-Y-SC-41).

\section{Publisher's Note}

Springer Nature remains neutral with regard to jurisdictional claims in published maps and institutional affiliations.

Received: 2 January 2019 Accepted: 26 March 2019

Published online: 30 April 2019 


\section{References}

1. Chinese Pharmacopoeia Commission. Chinese Pharmacopoeia. Shanghai: Science and Technology Press of Shanghai; 2015. p. 38. (in Chinese)

2. Anonymous. Chinese Pharmacopoeia, 1953 ed. 341. Beijing: China Medical Science and Technology Press; 1953. p. 24. (in Chinese).

3. Anonymous. Chinese Material Medica. 8. Shanghai: Science and Technology Press of Shanghai; 1999. p. 439-648. (in Chinese).

4. Anonymous. Chinese Pharmacopoeia, 2010 ed. 1. Beijing: China Medical Science and Technology Press; 2010. p. 24 (342-343). (in Chinese).

5. Anonymous. Chinese Pharmacopoeia, 1963 ed. 341. Beijing: China Medical Science and Technology Press; 1963. p. 24. (in Chinese)

6. Anonymous. Dictionary of Chinese Materia Medica. Shanghai: Science and Technology Press of Shanghai; 1977. p. 2525-8. (in Chinese)

7. Anonymous. Dictionary of Chinese Materia Medica. Shanghai: Science and Technology Press of Shanghai; 2015. p. 2525-8. (in Chinese).

8. Dao RL. Holy man's secret prescription for rehabilitation and injury disruption. Beijing: People's Health Publishing House; 1957. p. 78.

9. Anonymous. Dictionary of Chinese Materia Medica. Shanghai: Science and Technology Press of Shanghai; 2015. p. 595-1676. (in Chinese)

10. Zhang $\mathrm{H}, \mathrm{Hu} \mathrm{ZH}$. An developmental anatomical secondary structures in root of medicinal plants of Cyathula officinalis Chuan. Wuhan Inst Bot. 1998;22(3):219-20.

11. Zhao HJ, Shu GM, Zhou XJ, Bin XY, Li QM, Tian ML. Investigation of resource distribution and produced process of Cyathula officinalis Kuan in China. Resour Dev Mark. 2012;28(5):pp. 414-4, 471.

12. Liu W, Pei J, Yang M, Zhang YN. Discussion on origin change reason of Sichuan genuine crude drugs Cyathulae Radix. Chin Tradit Herb Drugs. 2016;47(9):1625-8.

13. Kuan KC, Xie ZW, Mao HX. Study on the botanical origin of the Chinese drug chuanniuxi and maniuxi. J Univ Chin Acad Sci. 1976;14(1):60-4.

14. Zhao HJ, Shu GM, Zhou XJ, Bin XY. Investigation of resource distribution and produced process of Cyathula officinalis Kuan in China. Resour Dev Market. 2012:28(05):414-6.

15. Wu XL. Identification of Cyathula officinalis Kuan and Cyathula capitata (wall.) Moq. J Guiyang Coll Tradit Chin Med. 2000;22(1):62-4.

16. Tian ML, Guan Y, Liu F, et al. Authentification of Radix Cyathule and its adulterants based on SCAR markers. China J Chin Mater Med. 2010;35(8):953-6.

17. Shang FQ. Comparative study on Achyranthese bidentata BI and Cyathula officinalis Kuan. Graduate School of Chinese Academy of Sciences (Wuhan Botanical Garden). 2016.

18. Zhou R, Li BG, Zhang GL. Chemical study on Cyatthula officinalis Kuan. J Asian Nat Prod Res. 2005;3:245-52.

19. Zhou R, Li BG, Zhang GL. Glycosides from roots of Cyatthula officinalis Kuan. J Integr Plant Biol. 2005;3:368-74.

20. Montoya G, Arango GJ, Ram RPJR. Rapid differentiation of isobaric and positional isomers of structurally related glycosides from Phytolacca bogotensis. Mass Spectrom. 2009;21:3361-71.

21. Zhao BT, Su YJ, Dong CM, Kun HS, Jong KS, Mi HW. High performance liquid chromatography used for quality control of Achyranthis Radix. Arch Pharmacal Res. 2012;35(8):1449-55.

22. Gu XH, Liu AX, Cheng Y, Xia YT, Han W. Extraction of polysaccharide from Cyathula officinalis Kuan. Biol Process. 2015;13(06):75-80.

23. Tong K, Li ZL, Sun X, Yan S, Jiang MJ, Deng MS, Chen J, Li JW, Tian ML. Metabolomics approach reveals annual metabolic variation in roots of Cyathula officinalis Kuan based on gas chromatography-mass spectrum. Chin Med. 2017;12:12

24. Zhao JJ, Yue YH, Xie Y, Liu LW, Cao F, Gao SR, Wang YJ. Radix Cyathula officinalis Kuan inhibits arterial remodeling in spontaneously hypertensive rats. Exp Ther Med. 2017;14:5395-400.

25. Chen H, Shi SH. Effects of Chinese herbs Radix Cyathulae and Radix Achyranthis Kuan Bidentatae on microcirculation and hemorheology of rats. China Microcirc. 1998;(3):182-4.

26. Wang YF, Kung HF, Gao D, Huang GM, Liu SJ. Effects of Si Junzi with Radix Cyathulae prescription on the healing of refractory wounds of lower limbs in rats. J Shanghai Univ Tradit Chin Med. 2013;27(03):95-8.

27. Wang YF, Li SJ, Yang WP, Gao D. Kung Huafa. Effects of Si Junzi with Radix Cyathulae prescription on fibroblasts and related cytokines in rats with refractory lower extremity wounds. J Shanghai Univ Tradit Chin Med. 2017:31(02):80-4
28. Qi M, Xin G, Zhu GQ. Effect of alcohol extract of Radix Cyathulae on blood pressure, myocardial ACE activity and myocardial cell diameter in spontaneously hypertensive rats. Mod Chin Med. 2010;12(06):34-7.

29. Liu TZ, Liu ZK. Radix Achyranthis Kuan and cortex moutan in the treatment of hypertension. Chin Med Mag. 2004;05:331.

30. Ding WM, Chen K, Li JG. Effects of Radix Cyathulae Decoction and polysaccharides on hepatocytes injured by $\mathrm{H}_{2} \mathrm{O}_{2}$ in rats. J Yangzhou Univ. 2013;34(04):11-4

31. Wang Y, Zhou GJ, Yan ZX, Kang HS, Chen YH, Chen M. Osteoprotective effect of Radix Achyranthis Kuan in ovariectomized rats. Chin J Osteopor. 2015:21(08):918-21.

32. Wang Y, Zhou GJ, Yan ZX, Kang HS, Chen YH, Chen M. Effects of Radix Achyranthis Kuan on estrogen and lipid metabolism in ovariectomized rats. China Matern Child Health. 2015;30(29):5063-6.

33. Wei S, Que HF. Study on the mechanism of Sanmiao Pills and Radix Achyranthis Kuan on inflammatory reaction in rats with acute gouty arthritis. World Tradit Chin Med. 2013;8(02):189-93.

34. Feng HB, Du XG, Liu J, Han XF, Cao XH, Zeng XY. Novel polysaccharide from Radix Cyathulae officinalis Kuan can improve immune response to ovalbumin in mice. Int J Biol Macromol. 2014:65:121-8.

35. Li ZL, Shi SH, Chen H, Liu YP. The immune activity of polysacharide of chuanniuxi. J Chin Med Mater. 1998:21(2):90-2.

36. Wang J, Fu T, Pu G, He KZ, Zhang GL. Study on the in vivo immune activity of polysaccharides from Radix Achyranthis Kuan. Pharmacol Clin Pract Tradit Chin Med. 2007;06:31-3.

37. Wang J, Pu T, He KZ, Fu T, Zhang GL. Study on the in vitro immune activity of Polysaccharides from Radix Achyranthis Kuan. Chin J Appl Environ Biol. 2008;14(4):481-3.

38. Song J, Yang JR, Li ZL. The inhibitory effect of Radix Achyranthis Kuan polysaccharide on mouse hepatoma cells. Sichuan J Physiol Sci. 2002:24(3):118-9.

39. Chen H, Liu YP. Preliminary study on the antitumor effect of Radix Achyranthis Kuan polysaccharide. J Chengdu Univ Tradit Chin Med. 2001;24(1):49-50

40. Zong CH, Guo XM, Han CP, Wang GY, Chu YH, Tian LM, Song GC, Bao $\mathrm{HH}$, Zhang SQ. Caspase-3 in Radix Achyranthis Kuan polysaccharideinduced apoptosis of hepatoma cell BEL-7402. J Mudanjiang Norm Univ. 2014;(3):31-2

41. Cao J, Wu L, Li K. Effects of Radix Achyranthis Kuan polysaccharides on tumor microenvironment T cell subsets, VEGF and TGF-beta1 in HepG-2 tumor-bearing mice. Appl Mod Chin Med. 2014;8(24):1-3.

42. Ding WM, Chen K, Li JG. Effects of Radix Achyranthis Decoction and polysaccharides on hepatocytes injured by $\mathrm{H}_{2} \mathrm{O}_{2}$ in rats. J Yangzhou Univ. 2013:34(04):11-4

43. Li JL, Han XF, Liu TQ, Zeng XY. Antioxidant effect of Radix Achyranthis Kuan polysaccharide on aging mice in vivo. Chin J Antibiot. 2014;39(07):553-9.

44. Chen Q, Xia YP, Qiu ZY. Effect of ecdysterone on glucose consumption in HepG2 cells. Chin Pharmacol Bull. 2005;21(11):1358-62.

45. Chen Q, Xia YP, Qiu ZY. Effects of ecdysterone on insulin sensitivity and glucose metabolism in insulin resistant cell model. Chin Pharmacol Bull. 2006;22(4):460-4

46. Wang JX, Sun M, Yang ZM, Li B, Liu Y, Zhang JZ. Comparison of moisture, ash and the whole extract of Yi medicine of Radix et Rhizoma Cyathulae Capithulae Capitata with Radix et Rhizoma Cyathulae officinalis and Radix et Rhizoma Achyranthis Bidentatae. Med Plant. 2013;4(Z1):27-30.

47. Pan J. The use of HPLC method for the determination of cyasterone in rabbit plasma. Chin J Conval Med. 2010;19(7):657-9.

48. Tang SQ, Chen $\mathrm{YH}$, Chen XP, et al. In vivo effect of guiding-herb Radix platycodonis and Radix cyathulae on paeiniflorin pharmacokinetics of Xuefu Zhuyu Tang in rats. Afr J Tradit Complement Altern Med. 2017;14(4):289-96.

49. Nanjing University of Traditional Chinese Medicine. Traditional Chinese medicine dictionary. Shanghai: Shanghai Science and Technology Press; 2006. p. 1020. ISBN 9787532382712.

50. Song SL. Experimental study on the pregnancy of mice in the decoction of Radix cyathulae. Chin J Med. 1994;9(4):232.

51. Bing XY. Pharmacological action of Radix cyathulae and its confused products. Luzhou: Luzhou Medical College; 2013. p. 12-4. 
52. Institute of Chinese Materia Medica. Chinese Academy of traditional Chinese medicine. Integrated TCM processing experience. Beijing: People's Medical Publishing House; 1974.

53. Tong K, Li ZL, Yan S, et al. HPLC fingerprint of crude Cyathula officinalis and its processed products stir-fried with wine and salt and variation of their index components. Chin Tradit Herb Drugs. 2016;47(4):580-4.

54. Zhou YY, Jiang M, Li W. Textual research of efficacy of Huainiuxi and Chuanniuxi. Pharm Clin Chin Mater Med. 2015;6(5):44-8. 